\title{
The Development of Guzheng Playing Technique and Its Application in Teaching
}

\author{
Nan Wu \\ College of Art, Xi'an University of Arts and Science, Xi'an, Shaanxi 710065, China \\ nan_wu83725@163.com
}

Keywords: Guzheng performance techniques, Development, Music teaching

\begin{abstract}
As one of the oldest and most distinctive ethnic musical instruments in our country, Guzheng not only can express the magnificent pride of imposing passion but also can play beautiful melody. Therefore, Guzheng playing has also become an important part of our musical culture. Guzheng teaching has also become an important part of our music teaching. However, with the enhancement of people's aesthetic demands for music, the traditional technique of playing Guzheng obviously can no longer meet the needs of the development of the times. This requires that Guzheng should learn new elements of the times, and it should innovate and develop continuously. After the undue efforts of Guzheng players and researchers, the present Guzheng playing has inherited the traditional techniques and has undergone relevant innovations and evolutions, presenting a diversified trend. At the same time, this development of Guzheng playing technique has been fully utilized to the music teaching. This article explores and thinks about it, hoping to promote the further development of the teaching of Guzheng playing.
\end{abstract}

\section{Introduction}

Guzheng handed down from Qin Dynasty, and has been more than two thousand years of history. In the following hundreds of years, through the creation and development of ancient literati and entertainers, Guzheng has continuously enriched and perfected their performance. ${ }^{1}$ In the past two thousand years, Guzheng techniques evolved a development process from single to diverse, from simple to complex. Guzheng itself takes Qin code as the boundary, and the strings are divided into two regions. "Right hand bomb, left hand press" has become the traditional statutory rules. In the traditional Guzheng playing process, left hand can only stay in the left side of the piano code, and works as the role of "retouch" right hand pronunciation, and is not involved in direct pronunciation. The innovative, complicated and diversified playing techniques have injected new vitality into the art of Guzheng. With the development of society and culture, people's pursuit of art has been enhanced. ${ }^{2}$ The original form of music has been unable to meet people's demand for art. This has also prompted the development of ancient music to break the tradition and innovate to meet the requirements of the development of the times. ${ }^{3}$

At this stage, although the technique of Guzheng has been combined with many harmony techniques, such as harmony and polyphony in the west, and its inherent national temperament is stable. Like a never-ending stream, new ingredients are continuously injected along the way, but its source can always be found. ${ }^{4}$ Traditional rhyme complementing sound can be inherited, and "rhyming and merging" is the same direction of the development of Guzheng music in the new era. Throughout the traditional musical instrument technology development process, the general development trend of music art is from mono to multi-sound, from simple to complex. ${ }^{5}$

Based on the above, this article will take time chronology as clues, and analyzes important works with detail. It always combined with the social and cultural background and other aspects of the omni-directional interpretation, and made a reasonable and scientific summary for Guzheng art development process of major events and phenomena. In the text the spectrum examples, form and text were also joined to illustrate my point of view, as far as possible clear thinking, clarity. 


\section{The Development of Guzheng Playing Techniques}

With the deepening of the internationalization of music art, based on the inheritance of traditional techniques, the technique of playing Guzheng also began to absorb new skill elements continuously to make it show new artistic features and development trend, and to study the development of the technique of playingGuzheng. Objectively speaking, the development of the era calls for the richer connotation of Guzheng music and the more variety of melody, which prompts the development of ancient Guzheng playing techniques to reach a new height. Therefore, based on many years of research experience, the author summarizes the following characteristics of the development of Guzheng performance.

\section{The Traditional Playing Techniques of Inheritance and Innovation}

At present, although the technique of playing Guzheng in our country incorporates many modern elements, the traditional playing technique still occupies an important position. In the performance of Guzheng, more traditional techniques such asclicking, kneading, chanting and trembling are used. These traditional techniques have condensed the essence of Guzheng playing technique and played an extremely important role in Guzheng playing. However, on the other hand, the traditional techniques have larger regional characteristics, which obviously do not meet the current needs of diversified music arts. ${ }^{6}$ Therefore, Guzheng playing skills must be based on the succession of targeted reform and innovation. the current Guzheng playing focuses on regulating the basic performance of hands and poses, and on the development of the left-handed play techniques, which all make Guzheng playing more flexible.

A kind of finger-playing method pioneered by Zhao Manqin in late 1979 was to use the principle of symmetry and the inertia of fingers to play. Because this method is very scientific and can meet the requirements of modern performance, it rises rapidly and becomes a major method of playing Guzheng. This method of performance broke the traditional method of playing before, with the use of four fingers other than the little finger to perform. Unfortunately, each finger can perform a separate performance, and the fingers can be matched accordingly. Therefore, the performance of Guzheng has great significance for us to use this method to play the fifth and seventh scales which can not be played by the traditional methods. As a result, the tunes of Guzheng can be broadened and become an important part of Guzheng's transformation from traditional instruments to modern instruments. Such as Guzheng music "Jing Gang Shan Shang Tai Yang Hong" was performed on the use of this quick fingering performance method.

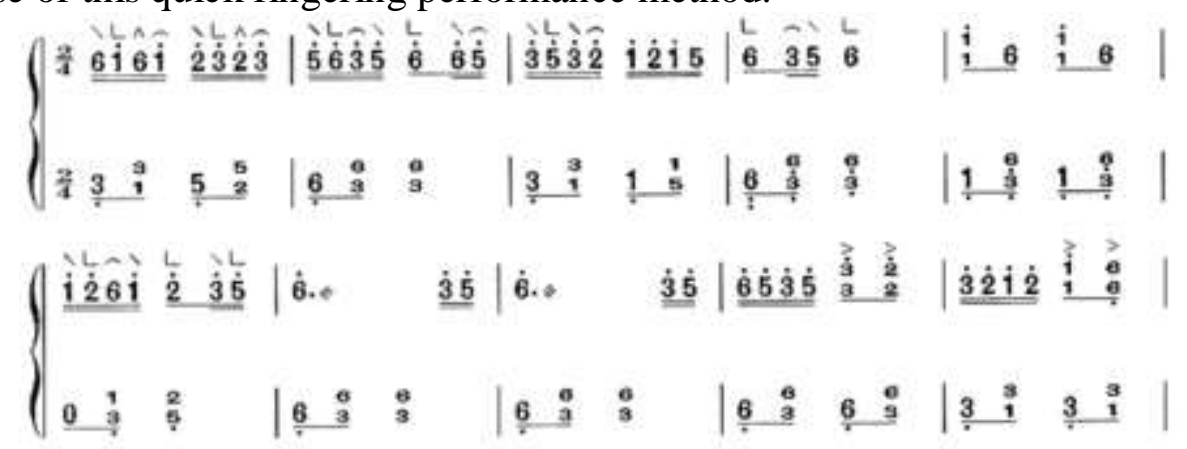

\section{Learn from Other Musical Instruments to Absorb Playing Skills}

The production and development of the technique of playing Guzheng are based on the characteristics of musical instruments and the law of musical development. On the basis of this, the absorption and use of other musical elements for integration make Guzheng more inclusive and rich. On the one hand, the absorption of other musical instruments byGuzheng is reflected in the reference to the national musical skill. For example, learn the strings of strokes Pipa and the percussion techniques of wooden fish. On the other hand, it is the fusion of western musical instrument playing techniques. For example, use fingers other than the middle finger, forefinger and thumb to participate in performance, and this gives full play to the function of the left hand in performance.

The Playing Style of The Diversified Development Trend

In addition to inheriting and innovating the traditional playing skills, the current Guzheng 
playing technique also absorbs and integrates into other musical instrument playing skills. At the same time, based on this, it presents a diversified development trend. On the basis of the traditional skills, such as clicking, rubbing, trembling, chanting, the techniques of turning the wheel, rocking the finger, pointing the finger and transforming the soundtrain are also formed to make Guzheng play an unprecedented vitality. On the basis of inheriting the national characteristics, the technique of playing Guzheng is more in line with the current characteristics of the current internationalization of music art.

Such as multi-finger skill: Guzheng song by Wang Zhongshan "Qiu Wang":

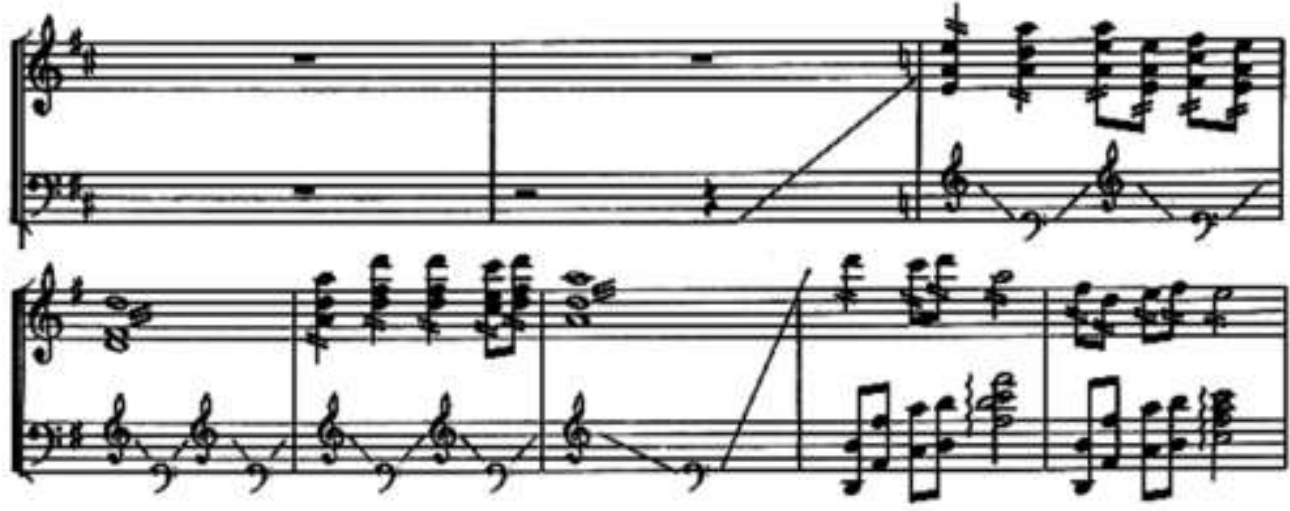

This section of music staff is the end of the music part of the composition. It usees multi-finger rocker technique, and reproducts the main theme. It makes the main melody from the monophonic melody into a rich and fluid and harmonic color band, so that the main melody sound effect is fuller, so as to make the theme image and music image further deeply and sublimate. This is not only the result of the ever-improving and expressive power of the music art form itself, but also the objective need of reflecting the real life evolving constantly.

\section{The Development of The Technique of Playing Guzheng in Music Teaching}

As the foundation and driving force of Guzheng art, Guzheng education not only provides the source of power for Guzheng playing, but also adjusts continuously with the development of Guzheng playing technique. Therefore, the development of Guzheng's playing technique is bound to be applied and reflected in music teaching, specifically, which mainly reflected in the following areas.

\section{Understand the Characteristics and Development of Guzheng's Musical Instruments}

First of all, the innovation of Guzheng playing technique requires that teachers should guide students to fully understand the characteristics and development of Guzheng's instruments. Grasp the fixed strings, left and right-hand gestures, phonemes, basic fingering and other basic theories and techniques, and have practice training based on these. During training, teachers should pay special attention to the correct posture and steps of the left and right finger movements when the students are playing. And teachers should explain to students in advance the more common fingering problems and causes, and put forward corresponding preventive and corrective measures. Instruct students to master the correct fingering and gestures to ensure the sound and speed of the play.

\section{Have a Solid Basic Skills}

Secondly, during the teaching of Guzheng music, teachers should instruct students to master solid basic skills through constant training and adjustment so that students can fully demonstrate the emotion and mood of different melodies through understanding the speed, strength and thickness of syllables. When instructing students to conduct Guzheng training, teachers should formulate corresponding training plans in light of the characteristics of "review tracks" and "new study tracks". For the "new learning track", teachers should guide students to slow down, even for fast track, which is also slow from the beginning of training. In addition, for those who are more difficult, more complex tricks of the tracks or fragments, to sub-chapters, students should practice step by step. In the chapters after the practice of re-integration, students should break the heavy training 
difficulties one by one. In learning new music at the same time, teachers should also arrange the students having a timely review of the tracks which have been learned, so that the new knowledge can be learnt. And teachers should guide students to summarize the playing techniques and rules based on the familiar tracks, and test them according to the type of new tracks.

\section{Strengthen Students Playing Training}

In addition, Guzheng teaching is as practical as other music teaching. Therefore, in addition to teaching basic theoretical knowledge, teachers should also require students to strengthen their playing training. Teachers should organize various evening parties, festivals, and other activities for students to provide a platform for presentation, so that students can continue to summarize and think through practice. Students can form the auditory and sensory communication, and gradually improve their cognitive standards of music.

\section{Summary}

The main emphasis of Guzheng during the performance is the audience's different pursuit of vision and hearing, the combination of music and body dancing, so that the audience can better understand the performers' feelings and psychology in the performance. Tish way of playing is more to attract the audience, but also can be a good slag pollution atmosphere, reflecting the player's playing strength. The relationship between this tradition and innovation is also essential to the succession of Guzheng music culture. As far as the current situation is concerned, Guzheng culture in our country has received good attention in the process of its development. However, in order to achieve greater success, it is necessary to handle the relationship between innovation and tradition and preserve the traditional fine culture of Guzheng. At the same time, it is better to conduct research on it and make bold innovations and developments.

\section{References}

[1] Fjellman-Wiklund, A.; Grip, H.; Karlsson, J. S.; Sundelin, G., EMG trapezius muscle activity pattern in string players: Part I-is there variability in the playing technique? International Journal of Industrial Ergonomics, 2004, 33 (4), 347-356.

[2] Flavin, P., The Koto: A Traditional Instrument in Contemporary Japan by Henry Johnson. Amsterdam: Hotei Publishing, 2004, 199 pp, no price given, hardback. British Journal of Music Education, 2005, 32 (2), 177-180.

[3] Lim, C. L., The current status of guzheng playing and its educational importance in Singapore schools. Journal of Obstetrics \& Gynaecology the Journal of the Institute of Obstetrics \& Gynaecology, 2007, 24 (5), 513-5.

[4] Shi, X. J.; Cai, Y. Y.; Chan, C. W., Electronic Music for Bio-Molecules Using Short Music Phrases. Leonardo, 2007, 40 (2), 137-141.

[5] Liu, L., Development and Application of Audio-triggered Courseware in Remote Music Teaching. International Journal of Emerging Technologies in Learning, 2015, 10 (6), 37.

[6] Schenker, H., The Technique of Playing the Piano. Art of Performance, 2002, 7-19. 